\title{
Individual Channel Estimation Based on Blind Interference Cancellation for Two-Way MI MO Relay Networks
}

\author{
Xianwen He, Gaoqi Dou* and Jun Gao \\ Department of Communication Engineering, Naval University of Engineering \\ Wuhan, 430033 - China \\ [e-mail: hxw_minipaper@163.com hjgcqq@163.com gaojunnj@163.com] \\ *Corresponding author: Gaoqi Dou
}

Received November 19, 2017; revised February 27, 2018; accepted April 10, 2018;

published August 31, 2018

\begin{abstract}
In this paper, we investigate an individual channel estimation problem for multiple-input multiple-output (MIMO) two-way amplify-and-forward (AF) relay networks. To avoid self-interference during the estimation of the individual MIMO channels, a novel blind interference cancellation (BIC) approach is proposed based on an orthogonal preceding framework, where a pair of orthogonal precoding matrices is utilized at the source nodes. By designing an optimal decoding scheme, we propose to decompose the bidirectional transmission into a pair of unidirectional transmissions. Unlike most existing approaches, we make the practical assumption that the nonreciprocal MIMO channel and the mutual interference of multiple antennas are both taken into consideration. Under the precoding framework, we employ an orthogonal superimposed training strategy to obtain the individual MIMO channels. However, the AF strategy causes the noise at the terminal to be the sum of the local noise and the relay-propagated noise. To remove the relay-propagated noise during the estimation of the second-hop channel, a partial noise-nulling method is designed. We also derive a closed-form expression for the total mean square error (MSE) of the MIMO channel from which we compute the optimal power allocation. The simulation results demonstrate that the analytical and simulated curves match fully.
\end{abstract}

Keywords: Channel estimation, two-way relay network, orthogonal precoding, superimposed training

This work was supported by National Natural Science Foundation of China (Grant No. 61302099) and also supported by China Postdoctoral Science Foundation (Grant Nos. 2015T81107). 


\section{Introduction}

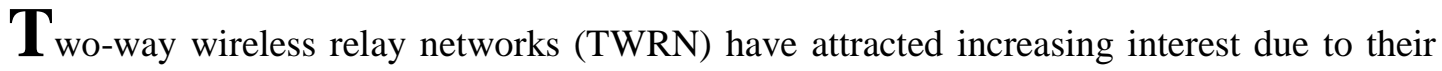
ability to increase network throughput and reduce the power consumption [1]-[2]. Multiple-input multiple-output (MIMO) techniques combined with TWRN can achieve significant multiplex gains and make full use the spatial domain with parallel independent data streams [3]-[6]. In [7]-[13], a number of advanced techniques were developed to optimize the MIMO relay network, such as optimal power allocation, relay precoding design, relay selection and linear transceiver design, all of which require accurate channel state information (CSI) of individual links in order to fully exploit the benefits of relay-aided networks. However, the CSI for different links is unavailable in practical relay networks and therefore, has to be estimated.

Unlike in traditional one-way relay networks, the receiver in two-way relay networks receives a superposition of the signal consisting of an unknown desired part and a known back-propagated part originating from itself, which is generally called self-interference [14] and must be removed by exploiting the estimated CSI prior to symbol detection. Recently, several training algorithms were developed to solve this problem. For the case of a single-antenna scenario, the schemes presented in [15]-[17] are very limited because it is not straightforward to extend them to multi-antenna scenarios due to the complicated constraint of the training design and the mutual interference of multiple antennas. Regarding the MIMO two-way relay network, Zhao et al. proposed a data-aided channel estimation algorithm that used the self-interference to obtain the coarse estimation of a MIMO channel matrix in [18]-[19]. In [20], Panah et al. investigated a pilot symbol-aided modulation where the composite and individual CSIs are estimated by exploiting the orthogonal pilots over the antenna arrays and the different terminals. In another study [21], the authors developed a channel estimation scheme for two-hop MIMO relay systems using a parallel factor analysis, which is applicable for both one-way and two-way relay networks with single or multiple relay nodes. In [22], the authors proposed two different estimation schemes consisting of a superimposed channel training scheme and a two-stage channel estimation scheme to obtain the individual MIMO CSI. It is noted that the contributions of [18]-[22] summarized the traditional interference cancellation framework and reported that the channel estimation must be performed prior to the interference cancellation. In this way, the equivalent channels of the TWRN comprised of the composite channels and the self-interfering channels are needed for the estimation. However, the residual self-interference due to an inevitable channel estimation error degrades the network performance and causes higher outage probabilities, bit error rates, etc. especially for MIMO relay networks; this was fully evaluated in [23]-[24].

In this study, we consider an individual channel estimation problem for MIMO two-way amplify-and-forward (AF) relay networks. To this end, we present a novel blind interference cancellation (BIC) scheme based on an orthogonal preceding framework, where a pair of orthogonal precoding matrices is utilized at the source nodes. In essence, the different orthogonal precoding matrices project the corresponding data onto the null space that is independent of the MIMO relay channel matrices [25]-[26]. By designing an optimal decoding scheme at the receiver, we can decompose the bidirectional transmission into a pair of unidirectional transmissions. Under the proposed precoding scheme, the self-interfering links and the information-bearing links are decoupled and the required complexity of the training design is greatly reduced. 
Unlike most existing approaches, we make the practical assumption that the nonreciprocal MIMO channel and the mutual interference of multiple antennas are both taken into consideration. Under the precoding framework, we employ the superimposed training strategy to obtain individual MIMO channels. First, to minimize the mean square error (MSE) of the composite MIMO channel, the optimal training sequences are designed with an orthogonal property not only over the antenna arrays but also between the source node and the relay node. However, the AF protocol causes the received noise at the terminal to be the sum of the local noise and the relay-propagated noise. Moreover, to remove the relay-propagated noise during the estimation of the second-hop channel, a partial noise-nulling (PNN) method is used at the relay node. Finally, we derive the closed-form expression for the total MSE of the individual MIMO channel and provide the optimal power allocation factor for the superimposed training at the relay node.

The remainder of the paper is organized as follows. The system model is introduced in Section 2 and the orthogonal precoding framework is designed in Section 3. The individual channel estimation for the proposed scheme is discussed in Section 4 and the optimal power allocation is presented in Section 5. The simulation results under different conditions are presented in Section 6 and the conclusions are provided in Section 7.

Notations: Boldface upper-case letters denote matrices and boldface lower-case letters denote vectors. The $N \times N$ identity matrix is denoted by $\mathbf{I}_{N}$. The superscripts $\mathrm{H}$, ${ }^{*}$, and $\mathrm{T}$ denote the complex conjugate transpose, complex conjugation, and transpose, respectively. $E\{\cdot\}$ is the expectation operator and vec $\{\cdot\}$ denotes the vectorization operator that stacks all column vectors of a matrix. The discrete Fourier transform (DFT) of a vector $\mathbf{x}$ is denoted by $\tilde{\mathbf{x}}=\mathbf{F}_{N} \mathbf{x}$, where $\mathbf{F}_{N}$ has $(m, n)$ entry $1 / \sqrt{N} e^{-j 2 \pi m n / N}$.

\section{System Model}

We consider a three-node MIMO-TWRN with an AF protocol shown in Fig. 1 including two source nodes $\mathbb{S}_{1}, \mathbb{S}_{2}$, and a relay node $\mathbb{R}$, where $\mathbb{S}_{1}$ and $\mathbb{S}_{2}$ are equipped with $N_{S}$ antennas and the relay node $\mathbb{R}$ has $N_{R}$ antennas. It is supposed that $N_{R} \geq N_{S}$, so that the network can support $N_{S}$ independent data streams as in [1]. In the first slot, both source nodes transmit the data blocks to the relay node. In the second slot, the relay node amplifies the received signals with a fixed gain and then broadcasts them to both source nodes. We assume that there is no direct link between $\mathbb{S}_{1}$ and $\mathbb{S}_{2}$. All of the nodes are assumed to operate in a half-duplex mode, i.e., the nodes cannot transmit and receive at the same time. The MIMO channels are assumed to exhibit flat Rayleigh fading and we assume that they do not change within the round for one frame transmission but vary for different frames.

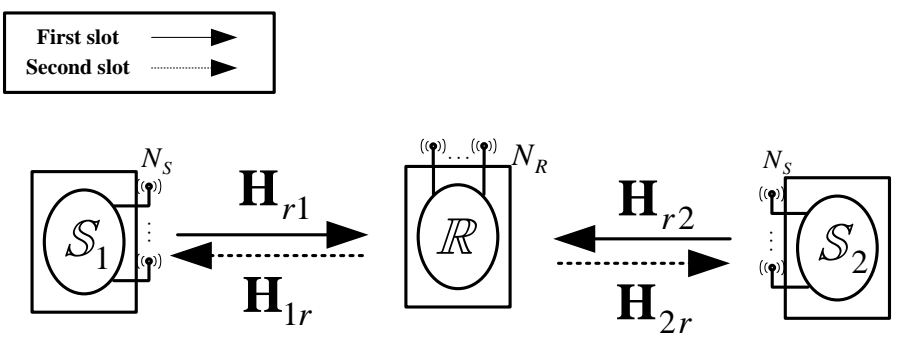

Fig. 1. Diagram of a two-way MIMO relay network with half-duplex antennas 
The source node considers a block-by-block transmission mode. As shown in Fig. 2, the first block in each frame is devoted to training. The block length is $N$ and the source training block is divided into $N_{S}$ sub-blocks with a fixed length $K\left(N=N_{S} K\right)$, which is denoted by $\mathbf{t}_{S}(n) \in \mathbb{C}^{K \times 1}, n=0,1, \cdots, N_{S}-1$. The $N_{S}$ sub-blocks are arranged in a source training matrix $\mathbf{T}_{S}=\left[\mathbf{t}_{S}(1), \mathbf{t}_{S}(2), . ., \mathbf{t}_{S}\left(N_{S}\right)\right]^{T} \in \mathbb{C}^{N_{S} \times K}, i \in 1,2$. The average transmission power at $\mathbb{S}_{i}$ is constrained by:

$$
P_{s}=\frac{\operatorname{tr}\left\{\mathbf{T}_{s} \mathbf{T}_{s}^{H}\right\}}{K}
$$

The key novelty is that a pair of precoding matrices $\mathbf{P}_{1} \in \mathbb{C}^{K \times M}$ and $\mathbf{P}_{2} \in \mathbb{C}^{K \times M}$ is employed at each source nodes, where $N_{S}$ output vectors of the precoded training can be represented in matrix form as:

$$
\mathbf{X}_{i}=\left[\begin{array}{c}
\mathbf{x}_{i}^{T}(0) \\
\mathbf{x}_{i}^{T}(1) \\
\vdots \\
\mathbf{x}_{i}^{T}\left(N_{s}-1\right)
\end{array}\right]=\mathbf{T}_{S} \mathbf{P}_{i} \in \mathbb{C}^{N_{S} \times M}, i=1,2
$$

Note that the precoder matrix $\mathbf{P}_{i}$ has full rank with the property $\operatorname{tr}\left\{\mathbf{P}_{i} \mathbf{P}_{i}^{H}\right\}=M, i \in 1,2$, which guarantees that the average power of the source data is unchanged after precoding [25]. In the first slot, the source nodes simultaneously transmit the data block to $\mathbb{R}$, where $\mathbf{x}_{i}^{T}(n), n=1,2, \ldots, N_{S}$ of $\mathbf{X}_{i}$ is serially transmitted over the $n$th transmitting antenna. The received signal at the relay node is faded due to the random MIMO channel and the relay noise is added, which is expressed as:

$$
\mathbf{R}=\mathbf{H}_{r 1} \mathbf{X}_{1}+\mathbf{H}_{r 2} \mathbf{X}_{2}+\mathbf{N}_{R}
$$

where $\mathbf{H}_{r 1} \in \mathbb{C}^{N_{R} \times N_{S}}$ and $\mathbf{H}_{r 2} \in \mathbb{C}^{N_{R} \times N_{S}}$ are the MIMO channel matrices from $\mathbb{S}_{1}$ to $\mathbb{R}$ and $\mathbb{S}_{2}$ to $\mathbb{R}$ respectively; their entries are independent and identically distributed as complex circular Gaussian random variables with a zero mean and the variances $\Omega_{1}$ and $\Omega_{2}$. $\mathbf{N}_{R} \in \mathbb{C}^{N_{R} \times M}$ is the noise matrix and its entries are the additive white Gaussian noise (AWGN) with a zero mean and the variance $\sigma_{R}^{2}$.

The relay $\mathbb{R}$ then amplifies the received signal $\mathbf{R}$ and superimposes its own training matrix as depicted in Fig. 2 . Therefore, the $N_{R} \times M$ signal matrix transmitted by the $\mathbb{R}$ can be denoted by:

$$
\mathbf{X}_{r}=\beta \mathbf{R}+\mathbf{T}_{R}\left(\mathbf{P}_{1}+\mathbf{P}_{2}\right)
$$

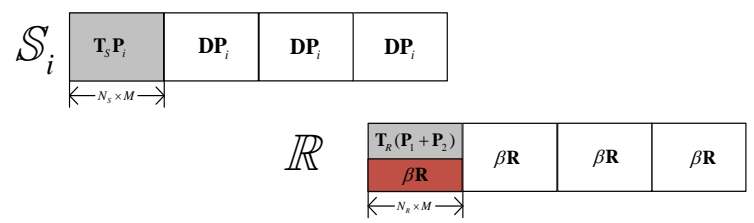

Fig. 2. Structure of the transmission frame using relay superimposed training 
where $\beta$ is the relay amplification factor (which is defined later) and $\mathbf{T}_{R}=\left[\mathbf{t}_{R}(1), \mathbf{t}_{R}(2), \ldots, \mathbf{t}_{R}\left(N_{R}\right)\right]^{T} \in \mathbb{C}^{N_{R} \times K}$ is the new relay training matrix. In the second slot, the relay broadcasts the resultant signal $\mathbf{X}_{r}$ to both source nodes under an average power constraint of:

$$
P_{r}=\frac{\operatorname{tr}\left\{\mathbf{X}_{r} \mathbf{X}_{r}^{H}\right\}}{M}
$$

We divide the average relay transmission power $P_{r}$ into two parts, where $\gamma P_{r}$ is allocated to the relay training matrix $\mathbf{T}_{R}$ and $(1-\gamma) P_{r}$ to the amplified received signal $\mathbf{R}$, where the power-allocation factor is satisfied by $0<\gamma<1$. The value of $\beta$ is given as:

$$
\beta=\sqrt{\frac{(1-\gamma) P_{r}}{N_{R}\left[P_{s}\left(\Omega_{1}+\Omega_{2}\right)+\sigma_{R}^{2}\right]}}
$$

Without loss of generality, we focus on signal processing at the source node $\mathbb{S}_{i}, i \in 1,2$. According to equations (3) and (4), the received signal at $\mathbb{S}_{i}$ is given as:

$$
\begin{aligned}
& \mathbf{Y}_{i}=\mathbf{H}_{i r} \mathbf{X}_{r}+\mathbf{N}_{i} \\
& =\underbrace{\left(\beta \mathbf{H}_{i r} \mathbf{H}_{r \bar{i}} \mathbf{T}_{S}+\mathbf{H}_{i r} \mathbf{T}_{R}\right) \mathbf{P}_{\bar{i}}}_{\text {desired signal part }}+\underbrace{\left(\beta \mathbf{H}_{i r} \mathbf{H}_{r i} \mathbf{T}_{S}+\mathbf{H}_{i r} \mathbf{T}_{R}\right) \mathbf{P}_{i}}_{\text {self-interference part }}+\beta \mathbf{H}_{i r} \mathbf{N}_{R}+\mathbf{N}_{i}
\end{aligned}
$$

where the elements in the matrix $\mathbf{N}_{i} \in \mathbb{C}^{N_{S} \times M}$ are also the AWGN as previously defined and $\mathbf{H}_{i r}$ are the MIMO channel matrices from $\mathbb{R}$ to $\mathbb{S}_{i}$ as previously defined ( $i=1,2$; i.e., $\bar{i}=1$ for $i=2$ and $\bar{i}=2$ for $i=1$ ).

In summary, the following assumptions are made for the transmission model.

(A1) The AWGN added at the relay node and the source node $\mathbb{S}_{i}$ are independent; each element has a zero mean and the covariance $\sigma_{R}^{2}$ or $\sigma_{i}^{2}$.

(A2) $\mathbf{H}_{i r}$ and $\mathbf{H}_{r i}$ are MIMO channel matrices; their entries are independent and identically distributed as complex circular Gaussian random variables with a zero mean and the variance $\Omega_{i}$.

(A3) The precoding matrix $\mathbf{P}_{i} \in \mathbb{C}^{K \times M}$ is full row-rank with $K<M$ and satisfies $\operatorname{tr}\left\{\mathbf{P}_{i} \mathbf{P}_{i}^{H}\right\}=M, \quad i=1,2$. This property guarantees that the average power of the source data is unchanged after precoding [25]-[26].

\section{Orthogonal Precoding Design}

The key novelty in our proposed BIC is that the training matrix $\mathbf{T}_{S}$ is postmultiplied by the specific precoding matrix $\mathbf{P}_{i}$; i.e., $\mathbf{X}_{i}=\mathbf{T}_{s} \mathbf{P}_{i}, i=1,2$, which means that each row vector $\mathbf{x}_{i}^{T}(n)$ of $\mathbf{X}_{i}$ is the linear combination of the row vectors of $\mathbf{P}_{i}$. From a vector-space perspective, the rows of the matrix $\mathbf{X}_{i}$ belong to the subspace spanned by the row vectors of the precoding matrix $\mathbf{P}_{i}$ [25]. Hence, each row of the desired received signal part in equation (7) also belongs to the row subspace of $\mathbf{P}_{i}$, which is independent of the unknown MIMO channel matrix [25]. As a result, the desired received signal part and the self-interference part 
can be decoupled by using different precoding matrices at both source nodes and by maintaining the orthogonal complement of the row subspace between $\mathbf{P}_{1}$ and $\mathbf{P}_{2}$. In order to cancel the respective self-interference and estimate the individual channel, a simple decoding strategy is designed at both source nodes with the decoding matrix $\mathbf{Q}_{i} \in \mathbb{C}^{M \times K}$; the resulting signal at the source node $\mathbb{S}_{2}$ is given by:

$$
\begin{aligned}
\mathbf{Y}_{i}^{Q} & =\mathbf{Y}_{i} \mathbf{Q}_{i} \\
& =\left(\beta \mathbf{H}_{i r} \mathbf{H}_{r \bar{i}} \mathbf{T}_{S}+\mathbf{H}_{i r} \mathbf{T}_{R}\right) \mathbf{P}_{\bar{i}} \mathbf{Q}_{i}+\left(\beta \mathbf{H}_{i r} \mathbf{H}_{r i} \mathbf{T}_{S}+\mathbf{H}_{i r} \mathbf{T}_{R}\right) \mathbf{P}_{i} \mathbf{Q}_{i}+\mathbf{N}_{e}
\end{aligned}
$$

where the equivalent noise matrix $\mathbf{N}_{e}=\beta \mathbf{H}_{i r} \mathbf{N}_{R} \mathbf{Q}_{i}+\mathbf{N}_{i} \mathbf{Q}_{i}$ and the decoding matrix $\mathbf{Q}_{i}$ satisfy the following properties:

$$
\mathbf{P}_{\bar{i}} \mathbf{Q}_{i}=\mathbf{I}_{K}, \quad i=1,2
$$

Then the decoding matrix $\mathbf{Q}_{i}, i=1,2$ can be obtained:

$$
\mathbf{Q}_{i}=\mathbf{P}_{\bar{i}}^{H}\left(\mathbf{P}_{\bar{i}} \mathbf{P}_{\bar{i}}^{H}\right)^{-1}, i=1,2
$$

On the other hand, to cancel the self-interference signal in equation (8), we design the decoder matrix $\mathbf{Q}_{i} \in \mathbb{C}^{M \times K}$ so that it is contained in the null space of $\mathbf{P}_{i}$, i.e.,

$$
\mathbf{P}_{i} \mathbf{Q}_{i}=\mathbf{0}_{K}
$$

Moreover, we consider maximizing the signal-to-noise ratio (SNR) in equation (8) and define the effective SNR as:

$$
\mathrm{SNR}=\frac{E\left\{\left\|\left(\beta \mathbf{H}_{i r} \mathbf{H}_{r \bar{i}} \mathbf{T}_{S}+\mathbf{H}_{i r} \mathbf{T}_{R}\right) \mathbf{P}_{\bar{i}} \mathbf{Q}_{i}\right\|\right\}}{E\left\{\left\|\mathbf{N}_{e}\right\|\right\}}
$$

One is then interested in finding the decoding matrix $\mathbf{Q}_{i}$ to maximize the above SNR, which is equivalent to designing the precoding matrix $\mathbf{P}_{\bar{i}}$. As a result, the problem is actually the minimization of the noise power $E\left\{\left\|\mathbf{N}_{e}\right\|\right\}$ with the optimal precoding matrix $\mathbf{P}_{\bar{i}}$, which is denoted by:

$$
\begin{aligned}
E & \left\{\left\|\mathbf{N}_{e}\right\|\right\} \\
& =\operatorname{tr}\left\{E\left\{\left(\beta \mathbf{H}_{i r} \mathbf{N}_{R}+\mathbf{N}_{i}\right) \mathbf{Q}_{i} \mathbf{Q}_{i}^{H}\left(\beta \mathbf{H}_{i r} \mathbf{N}_{R}+\mathbf{N}_{i}\right)^{H}\right\}\right\} \\
& =N_{S}\left(\beta^{2} N_{R} \Omega_{i} \sigma_{R}^{2}+\sigma_{i}^{2}\right) \operatorname{tr}\left\{\mathbf{Q}_{i} \mathbf{Q}_{i}^{H}\right\} \\
& =N_{S}\left(\beta^{2} N_{R} \Omega_{i} \sigma_{R}^{2}+\sigma_{i}^{2}\right) \operatorname{tr}\left\{\left(\mathbf{P}_{\bar{i}} \mathbf{P}_{\bar{i}}^{H}\right)^{-1}\right\}
\end{aligned}
$$

which is equivalent to minimizing $\operatorname{tr}\left\{\left(\mathbf{P}_{\bar{i}} \mathbf{P}_{\bar{i}}^{H}\right)^{-1}\right\}$ under the constraint condition in (A3). For an $L \times L$ positive definite matrix $\mathbf{M}$, we have $\operatorname{tr}(\mathbf{M}) \operatorname{tr}\left(\mathbf{M}^{-1}\right) \geq L^{2}$, where the equality holds if and only if $\mathbf{M}=\lambda \mathbf{I}_{L}$ for some nonzero constant $\lambda$. Using this and the fact that $\operatorname{tr}\left\{\left(\mathbf{P}_{\bar{i}} \mathbf{P}_{\bar{i}}^{H}\right)^{-1}\right\}$ is positive definite, $\mathbf{P}_{\bar{i}} \mathbf{P}_{\bar{i}}^{H}=(1 / \alpha) \mathbf{I}_{K}$ is satisfied when $\operatorname{tr}\left\{\left(\mathbf{P}_{\bar{i}} \mathbf{P}_{\bar{i}}^{H}\right)^{-1}\right\}$ is minimized and the constant $\alpha$ is defined later.

The following summarizes the relevant conditions regarding the precoding matrices and the decoding matrix.

(C1) According to equations (9) and (11), different precoding schemes at $\mathbb{S}_{i}$ are employed and we can guarantee that the subspaces spanned by the row vectors of $\mathbf{P}_{1}$ and $\mathbf{P}_{2}$ are orthogonal with: 


$$
\operatorname{span}\left\{\mathbf{P}_{1}\right\} \cap \operatorname{span}\left\{\mathbf{P}_{2}\right\}=\{\mathbf{0}\}
$$

where $\operatorname{span}\{\cdot\}$ stands for the linearly spanned space of the row vectors of a matrix.

(C2) The precoding matrix $\mathbf{P}_{\bar{i}} \in \mathbb{C}^{K \times M}$ is full row-rank under the constraint conditions that $\mathbf{P}_{\bar{i}} \mathbf{P}_{\bar{i}}^{H}=(1 / \alpha) \mathbf{I}_{K}, i=1,2$, where $\alpha=K / M$.

The optimal precoding and decoding matrices can be chosen from an arbitrary orthogonal matrix $\mathbf{O} \in \mathbb{C}^{M \times M}$ as follows:

$$
\begin{aligned}
& \mathbf{P}_{1}=\sqrt{\frac{M}{K}} \mathbf{O}(1: K,:) \in \mathbb{C}^{K \times M} \\
& \mathbf{P}_{2}=\sqrt{\frac{M}{K}} \mathbf{O}(K+1: M,:) \in \mathbb{C}^{K \times M}
\end{aligned}
$$

where $\mathbf{O}(i: j,:)$ denotes the $i^{\text {th }}$ to the $j^{\text {th }}$ rows of the orthogonal matrix $\mathbf{O}$ and $M=2 K$ is usually chosen for a symmetrical transmission procedure.

\section{Individual Channel Estimation}

\subsection{The optimal training design}

In our work, we consider the case of a nonreciprocal MIMO channel. Due to the self-interference operation with the decoding matrix, according to equation (8), the resulting signal at the source node can be rewritten in terms of an information-bearing cascaded MIMO channel $\mathbf{W}_{i \bar{i}}=\mathbf{H}_{i r} \mathbf{H}_{r \bar{i}}$ and a second-hop MIMO channel $\mathbf{H}_{i r}$ as

$$
\begin{aligned}
\mathbf{Y}_{i}^{Q} & =\mathbf{Y} \mathbf{Q}_{i} \\
& =\beta \mathbf{W}_{i \bar{i}} \mathbf{T}_{S}+\mathbf{H}_{i r} \mathbf{T}_{R}+\mathbf{N}_{e}
\end{aligned}
$$

Considering the structure of the received training signal block, a similar Least Square (LS) approach in a one-way relay network can be applied directly to estimate the individual MIMO channel matrices ( $\mathbf{H}_{i r}$ and $\mathbf{H}_{r \bar{i}}$ ). Accordingly, the received training block after vectorization can be denoted by:

$$
\begin{aligned}
& \mathbf{y}_{i}=\operatorname{vec}\left\{\mathbf{Y}_{i}^{Q}\right\} \\
& =\beta\left(\mathbf{T}_{S}^{T} \otimes \mathbf{I}_{N_{S}}\right) \mathbf{w}_{i \bar{i}}+\left(\mathbf{T}_{R}^{T} \otimes \mathbf{I}_{N_{S}}\right) \mathbf{h}_{i r}+\beta\left(\mathbf{Q}_{i}^{T} \otimes \mathbf{H}_{i r}\right) \mathbf{n}_{R}+\left(\mathbf{Q}_{i}^{T} \otimes \mathbf{I}_{N_{S}}\right) \mathbf{n}_{i}
\end{aligned}
$$

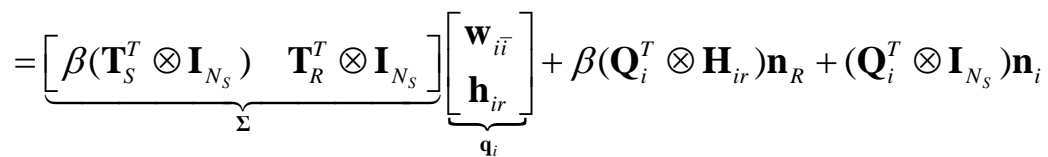

where $\mathbf{w}_{i \bar{i}}=\operatorname{vec}\left(\mathbf{W}_{i \bar{i}}\right), \mathbf{h}_{i r}=\operatorname{vec}\left(\mathbf{H}_{i r}\right), \mathbf{n}_{R}=\operatorname{vec}\left(\mathbf{N}_{R}\right), \mathbf{n}_{i}=\operatorname{vec}\left(\mathbf{N}_{i}\right)$. Here, the property of the vectorization operator that $\operatorname{vec}\{\mathbf{A B C}\}=\left(\mathbf{C}^{T} \otimes \mathbf{A}\right) \operatorname{vec}\{\mathbf{B}\}$ has been used to obtain equation (17). Due to its simplicity, the LS estimator is applied at the source node $\mathbb{S}_{i}$ to estimate $\mathbf{w}_{i \bar{i}}$ and $\mathbf{h}_{\text {ir }}$. We have:

$$
\hat{\mathbf{q}}_{i}=\boldsymbol{\Sigma}^{\dagger} \mathbf{y}_{i}
$$

The estimates $\hat{\mathbf{w}}_{i \bar{i}}$ and $\hat{\mathbf{h}}_{i r}$ are obtained from the corresponding vectors of $\hat{\mathbf{q}}_{i}$. According to $\mathbf{W}_{i \bar{i}}=\mathbf{H}_{i r} \mathbf{H}_{r \bar{i}}$, we note that the first-hop channel $\mathbf{H}_{r \bar{i}}$ may be expressed as: 


$$
\begin{aligned}
\hat{\mathbf{h}}_{r \bar{i}} & =\operatorname{vec}\left\{\hat{\mathbf{H}}_{r \bar{i}}\right\}=\operatorname{vec}\left\{\hat{\mathbf{H}}_{i r}^{\dagger} \hat{\mathbf{W}}_{i \bar{i}}\right\} \\
& =\left(\mathbf{I}_{N_{S}} \otimes \hat{\mathbf{H}}_{i r}^{\dagger}\right) \hat{\mathbf{W}}_{i \bar{i}}
\end{aligned}
$$

Using the MSE for the estimation of the individual MIMO channels is an obvious choice as a criterion for the optimal training design. However, the relationship between $\hat{\mathbf{h}}_{r \bar{i}}$ and $\hat{\mathbf{w}}_{i \bar{i}}$ is nonlinear, making it a big challenge to derive a closed-form expression for the MSE. However, the estimation accuracy of the individual MIMO channels is highly related to the quality of the estimation of $\mathbf{q}_{i}$. As a result, the MSE for the LS estimation of $\mathbf{q}_{i \bar{i}}$ is used as the criterion for the optimal training design with a closed-form expression. Under the relevant condition in $\mathbf{C 2}$, we have: $\mathbf{Q}_{i} \mathbf{Q}_{i}^{H}=\alpha \mathbf{I}_{M}$. The MSE of $\hat{\mathbf{q}}_{i}$ is directly given by:

$$
E\{\|\hat{\mathbf{q}}-\mathbf{q}\|\}=\alpha\left(\beta^{2} N_{R} \Omega_{i} \sigma_{R}^{2}+\sigma_{i}^{2}\right) \operatorname{tr}\left\{\left(\boldsymbol{\Sigma}^{H} \boldsymbol{\Sigma}\right)^{-1}\right\}
$$

where the expectation operation fully considers the noise and the MIMO channel statistics. A similar type of minimization was taken into consideration in [2] and [20]; we note that:

$$
\boldsymbol{\Sigma}^{H} \boldsymbol{\Sigma}=\left[\begin{array}{cc}
\beta^{2}\left(\mathbf{T}_{S} \mathbf{T}_{S}^{H} \otimes \mathbf{I}_{N_{S}}\right) & \beta\left(\mathbf{T}_{S} \mathbf{T}_{R}^{H} \otimes \mathbf{I}_{N_{S}}\right) \\
\beta\left(\mathbf{T}_{R} \mathbf{T}_{S}^{H} \otimes \mathbf{I}_{N_{S}}\right) & \mathbf{T}_{R} \mathbf{T}_{R}^{H} \otimes \mathbf{I}_{N_{S}}
\end{array}\right]
$$

Assuming that $\boldsymbol{\Sigma}$ is full-column rank, it is clear that $\boldsymbol{\Sigma}^{H} \boldsymbol{\Sigma}$ is the positive definite matrix. Based on the Cauchy-Schwarz inequality, we note that:

$$
\operatorname{tr}\left\{\left(\boldsymbol{\Sigma}^{H} \boldsymbol{\Sigma}\right)^{-1}\right\} \geq \sum_{i=1}^{N_{S}^{2}+N_{S} N_{R}} \frac{1}{\left[\boldsymbol{\Sigma}^{H} \boldsymbol{\Sigma}\right]_{i i}}
$$

where the MSE of $\mathbf{q}_{i}$ is minimized only if $\boldsymbol{\Sigma}^{H} \boldsymbol{\Sigma}$ is a diagonal matrix, i.e., the equality in equation (22) holds. Considering the mutual interference of multiple antennas in the MIMO system, the optimal training design should satisfy the following conditions:

$$
\begin{array}{ll}
\text { (C3) } \mathbf{T}_{R} \mathbf{T}_{S}^{H}=\mathbf{0} & \mathbf{T}_{S} \mathbf{T}_{R}^{H}=\mathbf{0} \\
\text { (C4) } \mathbf{T}_{S} \mathbf{T}_{S}^{H}=\frac{P_{S} K}{N_{S}} \mathbf{I}_{N_{S}} & \mathbf{T}_{R} \mathbf{T}_{R}^{H}=\frac{\gamma P_{r} K}{N_{R}} \mathbf{I}_{N_{R}}
\end{array}
$$

To design the optimal source training and relay training, we need at least $N_{S}+N_{R}$ mutually orthogonal vectors. Inspired by the training design in [18], the orthogonal vector groups may be generated from the $K \times 1$ single-root sequence $\tilde{\mathbf{c}}_{0}$ for $K \geq N_{S}+N_{R}$. After $N_{S}+N_{R}$ circular shifts for $\tilde{\mathbf{c}}_{0}$ in the frequency domain, we have the $K \times\left(N_{S}+N_{R}\right)$ circular matrix $\tilde{\mathbf{C}}_{0}$. We denote the circular orthogonal matrix as:

$$
\mathbf{T}=\sqrt{K} \mathbf{F}_{K}^{H} \tilde{\mathbf{C}}_{0}
$$

where $\mathbf{F}_{K}$ is the DFT matrix defined in the notations. Then, the optimal source training sequences are obtained from the first $N_{S}$ rows of the circular orthogonal matrix while the optimal relay ones are obtained from the next $N_{R}$ rows, i.e.,

$$
\begin{aligned}
& \mathbf{T}_{S}^{T}=\sqrt{\frac{P_{S}}{N_{S}}} \mathbf{T}\left(:, 1: N_{S}\right) \\
& \mathbf{T}_{R}^{T}=\sqrt{\frac{P_{r}}{N_{R}}} \mathbf{T}\left(:, N_{S}+1: N_{R}\right)
\end{aligned}
$$


With the selection of the training sequences, we guarantee that the conditions (C3)-(C4) are satisfied and that the MSE of $\mathbf{q}_{i}$ is minimized.

\subsection{Noise-nulling at the relay}

Because the relay noise is amplified by the relay-received signal according to equation (20), the noise at the receiver is the sum of the local noise and the relay-propagated noise; the latter has a serious effect on the MSE of the individual MIMO channel.

According to the optimal training design described in section 4.1, the source training and relay-training sequences are orthogonal to each other and occupy different pins in the frequency domain. In that case, we propose a method in which the partial noise is nulled at the relay.

To remove the relay-propagated noise during the estimation of the second-hop link, a PNN method is designed, where some special frequency components of the received training at the relay are discarded prior to training superimposition so that the relay-propagated noise added to the relay training is completely cancelled during channel estimation.

We assume that the indices of the non-zero pilot tones corresponding to $\mathbf{T}_{R}$ belong to $\mathcal{K}_{\mathrm{r}}$. A $K \times K$ frequency-domain diagonal matrix $\tilde{\mathbf{J}}=\operatorname{diag}\{\tilde{J}(0), \tilde{J}(1), \cdots, \tilde{J}(K-1)\}$ is defined by:

$$
\tilde{J}(k)= \begin{cases}1 & k \in \mathcal{K}_{r} \\ 0 & \text { otherwise }\end{cases}
$$

The corresponding diagonal matrix in the time domain is given by $\mathbf{J}=\mathbf{F}_{K}^{H} \tilde{\mathbf{J}} \mathbf{F}_{K}$. For a received training signal, the $M \times M$ noise-nulling matrix under the orthogonal preceding framework is given by:

$$
\mathbf{J}_{N}=\mathbf{Q}_{1}\left(\mathbf{I}_{K}-\mathbf{J}\right)^{T} \mathbf{P}_{1}+\mathbf{Q}_{2}\left(\mathbf{I}_{K}-\mathbf{J}\right)^{T} \mathbf{P}_{2}
$$

The relay first nulls the noise from the received vector $\mathbf{R}$ and then amplifies it before adding the relay-training matrix $\mathbf{T}_{R}$ on top of it. According to equation (4), the signal matrix transmitted by $\mathbb{R}$ using PNN method can be refreshed by:

$$
\overline{\mathbf{X}}_{r}=\beta^{\prime} \mathbf{R} \mathbf{J}_{N}+\mathbf{T}_{R}\left(\mathbf{P}_{1}+\mathbf{P}_{2}\right)
$$

where $\beta^{\prime}$ is the modified amplification factor which is reset as:

$$
\beta^{\prime}=\sqrt{\frac{(1-\gamma) P_{r}}{N_{R}\left\{P_{s}\left(\Omega_{1}+\Omega_{2}\right)+\left[1-N_{R} /\left(N_{S}+N_{R}\right)\right] \sigma_{R}^{2}\right\}}}
$$

\section{Optimal power allocation}

With the objective to minimize the total MSE of the $\mathbf{W}_{i \bar{i}}$ and $\mathbf{H}_{i r}$, the optimal power allocation between the relay training and the relay-received signal is formulated as:

$$
\begin{array}{ll}
\min _{\mathbf{P}_{i}, \mathbf{Q}_{i}} & M S E_{w_{i \pi}}+M S E_{h_{i r}} \\
\text { s.t. } & \text { (C3), (C4) }
\end{array}
$$

However, with the PNN method employed at the relay node, the MSE of $\mathbf{q}_{i \bar{i}}=\left[\mathbf{w}_{i \bar{i}}^{T}, \mathbf{h}_{i r}^{T}\right]^{T}$ is altered. According to equation (20), the MSE of $\mathbf{w}_{i \bar{i}}$ and the MSE of $\mathbf{h}_{i r}$ can be denoted by: 


$$
\begin{gathered}
\operatorname{MSE}_{\mathbf{h}_{\text {ir }}}=\frac{\alpha N_{S} N_{R}^{2} \sigma_{i}^{2}}{\gamma K P_{r}} \\
M S E_{\mathbf{w}_{i \bar{i}}}=\frac{\alpha N_{S}^{3}\left(\beta^{\prime 2} N_{R} \Omega_{i} \sigma_{R}^{2}+\sigma_{i}^{2}\right)}{\beta^{\prime 2} K P_{s}}
\end{gathered}
$$

According to equation (31), it is clearly noted that after PNN at the relay node, the MSE for the estimation of the second-hop MIMO channels is not affected by the relay-propagated noise. Substituting $M S E_{\mathbf{h}_{i r}}$ and $M S E_{\mathbf{w}_{\bar{i}}}$ into equation (30), we can verify that the optimal problem for the total MSE can be traced to the power-allocation factor $\gamma$. It is noted that, from a practical deduction, the objective function can be denoted by:

$$
\begin{aligned}
f(\gamma) & =\frac{\alpha N_{S}^{3}\left(\beta^{\prime 2} N_{R} \Omega_{i} \sigma_{R}^{2}+\sigma_{i}^{2}\right)}{\beta^{\prime 2} K P_{s}}+\frac{\alpha N_{S} N_{R}^{2} \sigma_{i}^{2}}{\gamma K P_{r}} \\
& =\frac{\alpha N_{S}^{3} N_{R} \Omega_{i} \sigma_{R}^{2}}{K P_{S}}+\frac{\alpha N_{S}^{3} N_{R} \chi \sigma_{i}^{2}}{(1-\gamma) P_{r} K P_{S}}+\frac{\alpha N_{S} N_{R}^{2} \sigma_{i}^{2}}{\gamma K P_{r}}
\end{aligned}
$$

where $\beta^{\prime}=\sqrt{(1-\gamma) P_{r} / N_{R} \chi}$ and $\chi=P_{s}\left(\Omega_{1}+\Omega_{2}\right)+\left[1-N_{R} /\left(N_{S}+N_{R}\right)\right] \sigma_{R}^{2}$. The first derivation can be expressed as:

$$
f^{\prime}(\gamma)=-\frac{\alpha N_{S}^{3} N_{R} \chi \sigma_{i}^{2}}{(1-\gamma)^{2} P_{r} K P_{s}}-\frac{\alpha N_{S} N_{R}^{2} \sigma_{i}^{2}}{\gamma^{2} K P_{r}}
$$

when $f^{\prime}(\gamma)=0$, we have

$$
N_{S}^{2} \chi \gamma^{2}+(1-\gamma)^{2} N_{R} P_{s}=0
$$

the optimal $\gamma$ can be derived as:

$$
\gamma_{1}=\frac{N_{R} P_{s}-N_{s} \sqrt{N_{R} P_{s} \chi}}{N_{R} P_{s}-N_{s}^{2} \chi}, \gamma_{2}=\frac{N_{S} \sqrt{N_{R} P_{s} \chi}+N_{R} P_{s}}{N_{R} P_{s}-N_{S}^{2} \chi}
$$

Obviously, we have $N_{R} P_{s}-N_{s}^{2} \chi<0$. As a result, $\gamma_{1}$ is the only effective solution.

\section{Simulation Results}

In this section, we present the simulation results to evaluate the proposed scheme with the fixed antenna parameters $N_{S}=N_{R}=2$. All the transmission nodes are assumed to have the same power constraint, i.e., $P_{s}=P_{r}$. The SNRs of the links are assumed as $\sigma_{n}^{2}=\sigma_{R}^{2}=\sigma_{i}^{2}=1$ and $S N R=P_{s} / \sigma_{n}^{2}=P_{r} / \sigma_{n}^{2}$. The MIMO channel is randomly generated and assumed to have uncorrelated Rayleigh fading. The variance of the MIMO channel matrix $\Omega_{i}=\mathcal{G} / d_{i}^{\varepsilon}$, where $d_{i}$ denotes the distances between $\mathbb{S}_{i}$ and $\mathbb{R}, \varepsilon=3, \mathcal{G}=1$. The relay node is assumed to be positioned in the middle between the two source nodes so that $d_{1}=d_{2}=1$. 


\subsection{Complexity Analysis}

To avoid self-interference during the estimation of the individual MIMO channels, we propose the BIC scheme. The key novelty is that a pair of precoding matrices is employed at each source nodes. Correspondingly, by designing an optimal decoding scheme at the receiver, we can decompose the bidirectional transmission into a pair of unidirectional transmissions. As a result, extra $K M$ number of multiplication is performed at the transmitting node and the receiving node respectively.

What's more, to remove the interference from the relay-propagated noise, we propose a method in which the partial noise is nulled at the relay. In theory, the DFT/IDFT operations are firstly needed for the received signal at the relay and then some frequency components of the received training signal are discarded to null the relay-propagated noise. However, nulling operations can be equivalently performed in time domain with low computational complexity in practice, even only addition/abstraction operations are requested in some cases. Comparing to the ST schemes in [22], only extra $M^{2}$ number of multiplication is performed at the relay node.

It is seen that as compared with the ST scheme in [22], the extra operations for the proposed scheme is small, which can be negligible as compared with complicated channel estimation for two-way relay networks.

\subsection{MSE performance}

As is shown in Fig. 3, we first investigate the estimation performance of the individual MIMO channels with different training designs. Consider that each training block consists of 8 training symbols, which are divided into $N_{S}=2$ blocks with a fixed length $K=4$ and arranged into a $2 \times 4$ source training matrix $\mathbf{T}_{S}$. Accordingly, a pair of precoding matrices $\mathbf{P}_{1}$ and $\mathbf{P}_{2}$ are of the size $4 \times 8(M=8)$ and are selected from a $8 \times 8$ Walsh-Hadamard matrix. We assume that the power allocation factor is $\gamma=0.1$ unless stated otherwise. For comparison, two kinds of training designs are taken into consideration: (1) a fully uncorrelated training design based on section 4.1, where the training designs are independent between the relay node and the source node and for all antennas, i.e., $\mathbf{T}_{S} \mathbf{T}_{R}^{H}=\mathbf{0}$; (2) a training design uncorrelated for all antennas but equal for the relay node and source node, i.e., $\mathbf{T}_{S}=\mathbf{T}_{R}$. It is clearly noted that the optimal training design has a better MSE performance because the interference between the source and relay training is completely cancelled. When more power is allocated to the relay-received signal, the cascaded MIMO channel (denoted by ' $\mathbf{W}$ ' in the figure) is more accurate than the second-hop MIMO channel (denoted by ' $\mathbf{H}_{i r}$ ' in the figure) estimator. As we can see, the PNN method provides a substantial improvement in the accuracy. In the scenario without PNN method, the relay simply adds its own training to the amplified version of the received training signal, where the relaying noise is also amplified and propagated through fading individual channel. Consequently, the equivalent noise is the colour noise, including the local AWGN and the amplified version of the propagated noise from the relay node, which degraded the estimation performance of individual channel ( $\left.\mathbf{H}_{\text {ir }}\right)$. To remove the relay-propagated noise during the estimation of the second-hop link, a PNN method is designed, where some special frequency components of the received training at the relay are discarded prior to training superimposition so that the relay-propagated noise added to the relay training is completely cancelled during channel estimation. The analytical 
performance (denoted by 'Theory' in the figure) is obtained from equations (20), (31), and (32). The simulation results closely approach the analytical MSE value.

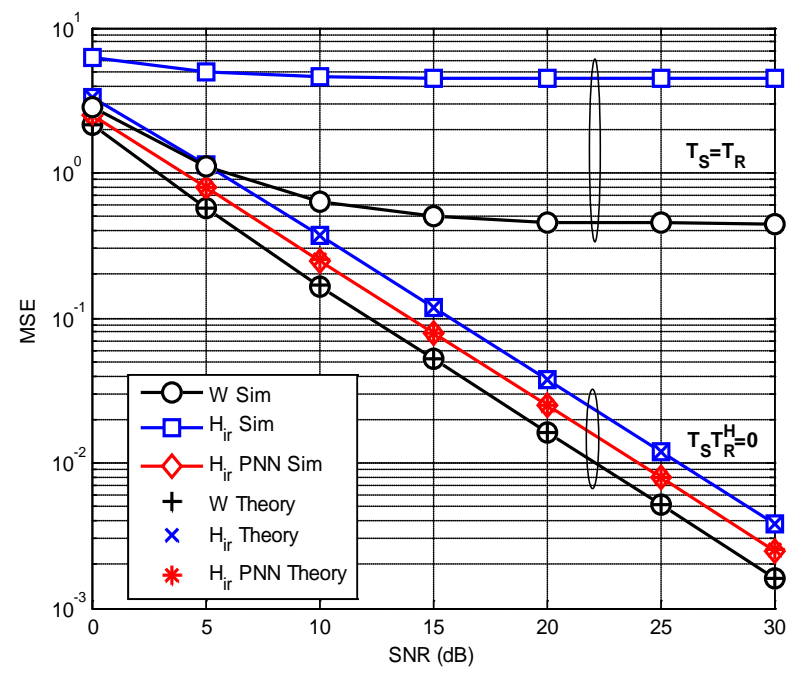

Fig. 3. MSE performance of the LS estimator using BIC with different training designs

As is shown in Fig. 4, we investigate the estimation performance of the individual MIMO channels with different training lengths $(K=4,8,16)$. It is clearly noted that both the MSE performance of the cascaded MIMO channel and the second-hop MIMO channel with the PNN method improve with an increasing number of the training length; this is consistent with the derivation of equations (31) and (32).

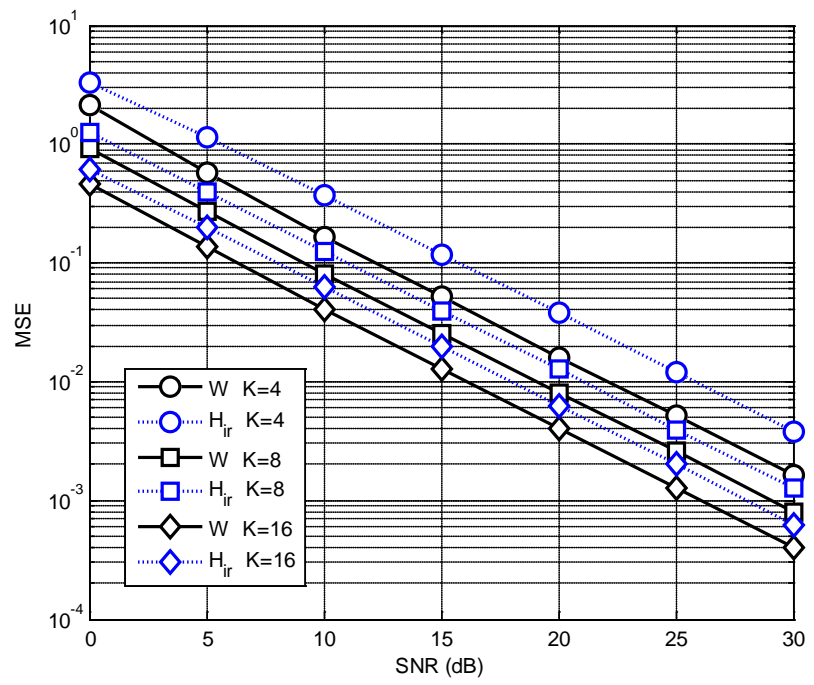

Fig. 4. MSE performance of the LS estimator using BIC with $K=4,8,16$ 
To investigate the validity of the BIC schemes, we compare them with the superimposed training (ST) scheme and two-stage scheme in [22] (denoted by "ST" and "Two-Stage" in the figures). In the simulation, we assume that the power allocation factor is $\gamma=0.1$ and the training length $K=4$ for all of the channel estimation schemes. The MSE of the individual channel estimation versus SNR is shown in Fig. $\mathbf{5}$. It is seen from Fig. 5 that the three schemes almost have the same performance of cascaded links ( $\mathbf{W}$ ). Moreover, it is clearly seen that due to the effect of relay propagated noise, the BIC scheme is much better than the ST scheme and almost the same with two-stage scheme in the MSE of individual links $\left(\mathbf{H}_{i r}\right)$. It is because that the relay simply adds its own training to the amplified version of the received training signal in ST scheme, where the relaying noise is also amplified and propagated through fading individual channel. Consequently, the equivalent noise is the colour noise, which degraded the estimation performance of individual channel $\left(\mathbf{H}_{\text {ir }}\right)$ in ST scheme. However in the two-stage scheme, both source nodes are silent at the first stage, while the relay node broadcasts the relay training to both receive node for the individual channel $\left(\mathbf{H}_{\text {ir }}\right)$ which is totally unaffected by relay propagated noise.

Actually, both the ST scheme and two-stage scheme can avoid self-interference during the estimation of the individual MIMO channels with optimal training design. However, the two-stage scheme has to occupy the extra communication slots which reduce the transmission efficiency seriously. As for ST scheme, besides the impact of the relay propagated noise, the complicated orthogonal constraint must be enforced, which implies the source node in relay networks not only has to preknow the information of the relay training but also the information of the source training of the other source node in order to design their own training sequences to maintain the special form of orthogonality. As a result, extra overheads are needed to coordinate the training design of different nodes. Finally, on the basis of above analysis, the proposed BIC scheme has obvious advantages.

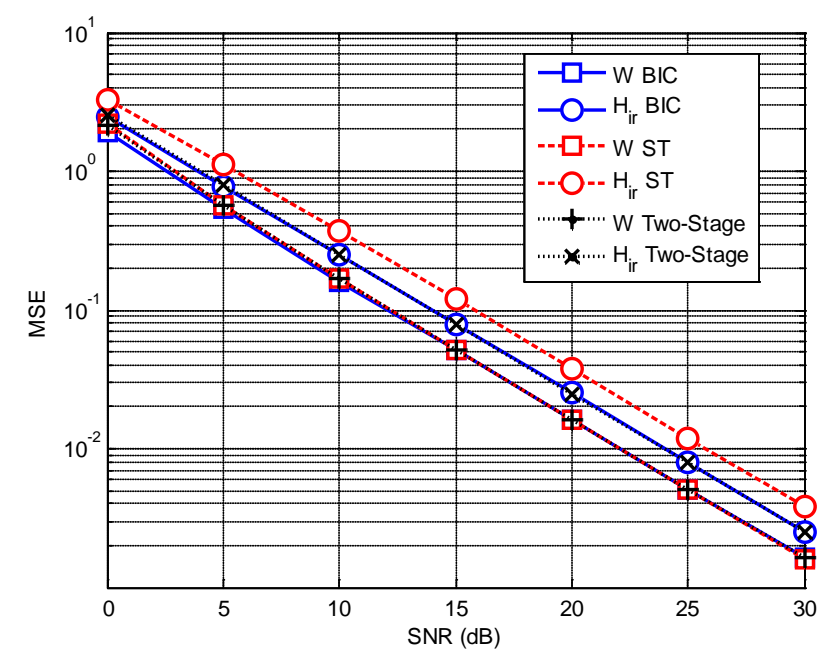

Fig. 5. MSE performance comparison between BIC scheme and other different schemes 


\subsection{Power allocation}

Fig. 6 shows the individual MSE performances using a PNN method when $S N R=10,20,30 d B$. As before, the training matrix has a size of $2 \times 4\left(N_{S}=2, K=4\right)$ while the pair of precoding matrices $\mathbf{P}_{1}$ and $\mathbf{P}_{2}$ are of size $4 \times 8(M=8)$. When more power is allocated to the relay training, the second-hop MIMO channel achieves a better estimation performance. In contrast, when less power is allocated to the relay-received source training signal, the cascaded MIMO channel estimator exhibits a worse performance.

As is shown in Fig. 6, the total MSE in equation (30) is obtained using the optimal training design with respect to the power-allocation factor $\gamma$ when $S N R=10,20,30 \mathrm{~dB}$. According to equation (36), there is an optimal value of the power-allocation factor that minimizes the total MSE. The derivation of the optimal values when $S N R=10,20,30 d B$ is shown in Table 1 It is evident in Fig. 6 that the analytical and simulated curves are perfectly matched for all SNRs and that the optimal power-allocation factor is insensitive to the $S N R$ when $S N R \geq 10 \mathrm{~dB}$.

Table 1. The optimal power-allocation factor with different SNRs

\begin{tabular}{|c|c|}
\hline SNR & The optimal power-allocation factor \\
\hline \hline $10 \mathrm{~dB}$ & $\gamma=0.3306$ \\
\hline $20 \mathrm{~dB}$ & $\gamma=0.3331$ \\
\hline $30 \mathrm{~dB}$ & $\gamma=0.3333$ \\
\hline
\end{tabular}

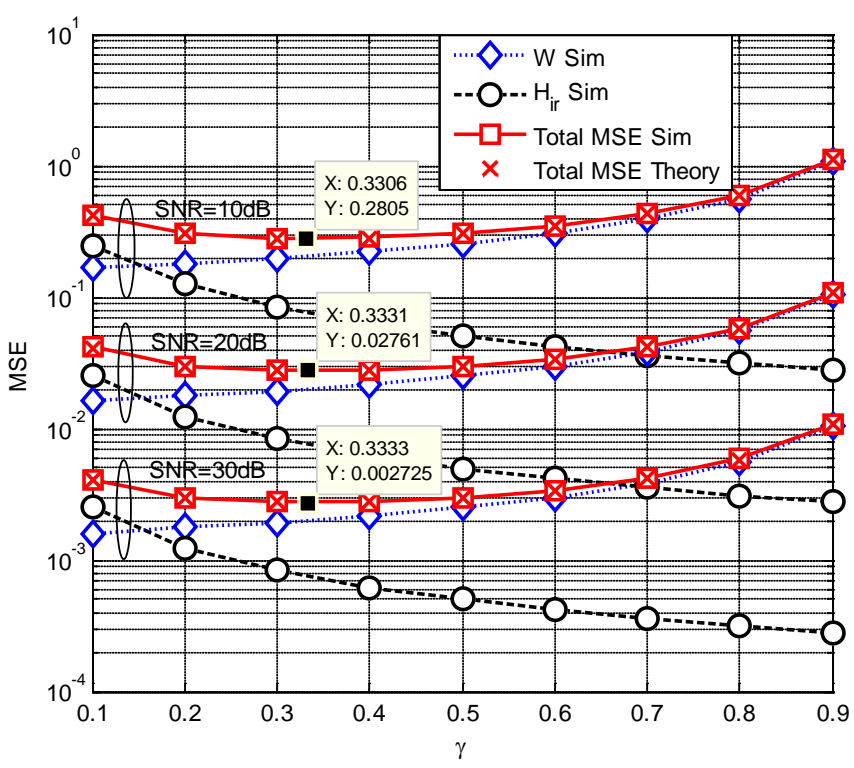

Fig. 6. MSE performance of the LS estimator using a PNN method for different power-allocation factors when $S N R=10,20,30 d B$

\section{Conclusion}

To remove the self-interference during the estimation of individual MIMO channels, we present a novel BIC scheme based on an orthogonal preceding framework, where a pair of orthogonal precoding matrices is utilized at the source nodes. Under the precoding framework, we use the orthogonal superimposed training strategy to obtain the individual MIMO channels. 
To remove the relay-propagated noise during the estimation of the second-hop channel, a PNN method is designed and the closed-form expression for the total MSE of the MIMO channel is derived. The optimal power allocation is obtained from the expression. The simulation results demonstrate that the PNN method provides a substantial improvement in accuracy for the second-hop MIMO channel estimation and that the analytical and simulated optimal power-allocation factors are fully matched.

\section{References}

[1] R. Wang, M. Tao and Z. Xiang, "Nonlinear precoding design for MIMO amplify-and-forward two-way relay systems, ” IEEE Transactions on vehicular technology, vol. 61, no.9, pp.3984-3995, November, 2012. Article (CrossRef Link).

[2] S. Abdallah and I. N. Psaromiligkos, "Semi-blind channel estimation with superimposed training for OFDM-based AF two-way relaying," IEEE Transactions on wireless communications, vol. 13, no. 5, pp. 2468-2477, May, 2014. Article (CrossRef Link).

[3] R. Wang, M. Tao, H. Mehrpouyan, et.al, "Channel estimation and optimal training design for correlated MIMO two-way relay systems in colored environment, " IEEE Transactions on wireless communications, vol.14, no. 5,pp. 2684-2699, May, 2015. Article (CrossRef Link).

[4] Z. He, Z. Lang, Y. Rong, et.al. "Joint transceiver optimization for two-way MIMO relay systems with MSE constraints,” IEEE Wireless Communication Letters, vol. 3, no.6, pp. 613-616, December, 2014. Article (CrossRef Link).

[5] C. Wang, J. Chen and Y. Peng. "Relay precoder designs for two-way amplify-and-forward MIMO relay systems: an eigenmode-selection approach," IEEE Transactions on wireless communications, vol. 15, no. 7, pp.5127-5137, July, 2016. Article (CrossRef Link).

[6] S. Devendra and K. Prabhat. "Impact of channel estimation error on zero-forcing based MIMO two-way relaying," IET Signal processing, vol. 10, no. 3, pp. 210-217, March, 2016. Article (CrossRef Link).

[7] K. Nguyen, Y. Rong and S. Nordholm. "Simplified MMSE precoding design in interference two-way MIMO relay systems,” IEEE Signal Processing Letters, vol. 23, no. 2, pp. 262-266, February, 2016. Article (CrossRef Link).

[8] M. Tao and R. Wang, "Linear precoding for multi-pair two-way MIMO relay systems with max-min fairness," IEEE Transactions on signal processing, vol. 60, no. 10, pp. 5361-5370, October, 2012. Article (CrossRef Link).

[9] C. Hu, G. Liu and B. Chen, "Joint relay/antenna selection and precoding design for two-way MIMO amplify-and forward relaying systems," IEEE Transactions on vehicular technology, vol. 65, no. 7, pp. 4854-4864, July, 2016. Article (CrossRef Link).

[10] J. You, E. Liu, R. Wang, et.at, "Joint source and relay precoding design for MIMO two-way relay systems with transceiver impairments," IEEE Communications letters, vol. 21, no. 3, pp. 572-575, March, 2017. Article (CrossRef Link).

[11] L. Sanguinetti, A. A. D'Amico and Y. Rong, “A tutorial on the optimization of amplify-and-forward MIMO relay systems”, IEEE J. Sel. Areas Communications, vol. 30, no. 8, pp. 1331-1346, September, 2012. Article (CrossRef Link).

[12] J. Kang and H. Kim, "Optimal training design for MIMO-OFDM two-way relay networks," IEEE Transactions on communications, vol. 65, no. 9, pp. 3675-3690, September, 2017. Article (CrossRef Link).

[13] F. Gao, R. Zhang and Y. C. Liang, "Channel estimation for OFDM modulated two-way relay networks," IEEE Transactions on signal processing, vol. 57, no. 11, pp. 4443-4455, November,2009. Article (CrossRef Link).

[14] B. Zhong and Z. Zhang. "Secure full-duplex two-way relaying networks with optimal relay selection”, IEEE Communications Letters, vol. 21, no. 5, pp. 1123-1126, May, 2017. Article (CrossRef Link). 
[15] H. Zhang, H. Xing, J. Cheng, et al. "Secure resource allocation for OFDMA two-way relay wireless sensor networks without and with cooperative jamming," IEEE Transactions on industrial informatics, vol. 12, no. 5, pp. 1714-1725, October, 2016. Article (CrossRef Link).

[16] G. Wang, F. Gao, Y, C. Wu, et.al, "Joint CFO and channel estimation for OFDM-based two-way relay networks,” IEEE Transactions on wireless communication, vol. 10, no. 2, pp. 456-465, February, 2011. Article (CrossRef Link).

[17] S. Zhang, F. Gao and C. X. Pei, "Optimal training design for individual channel estimation in two-way relay networks,” IEEE Transactions on signal processing, vol. 60, no. 9, pp. 4987-4991, September, 2012. Article (CrossRef Link).

[18] J. Zhao, M. Kuhn, A.Wittneben, et.at, "Self-interference aided channel estimation in two-way relaying systems," in Proc. of IEEE Global Telecommunication Conf., pp. 3659-3664, November 30-December 4, 2008. Article (CrossRef Link).

[19] J. Zhao, M. Kuhn, A.Wittneben, et.at, “Achievable rates of MIMO bidirectional broadcast channels with self-interference aided channel estimation,” in Proc. of IEEE Wireless Communication Netw. Conf. pp. 1-6, April 5-8, 2009. Article (CrossRef Link).

[20] A. Y. Panah and W. Heath, "MIMO two-way amplify-and-forward relaying with imperfect receiver CSI," IEEE Transactions on vehicular technology, vol. 59, no. 9, pp. 4377-4387, November, 2010. Article (CrossRef Link).

[21] Y. Rong, R. A. Khandaker and Y. Xiang, ”Channel estimation of dual-hop MIMO relay system via parallel factor analysis," IEEE Transactions on wireless communications, vol. 11, no. 6, pp. 2224-2233, June, 2012. Article (CrossRef Link).

[22] C. Chiong, Y. Rong and Y. Xiang. "Channel training algorithms for two-way MIMO relay systems,” IEEE Transactions on signal processing, vol. 61, no. 16, pp. 3988-3998, August, 2013. Article (CrossRef Link).

[23] A. Vosoughi and Y. Jia, "How does channel estimation error affect average sum-rate in two-way amplify-and-forward relay networks,” IEEE Transactions on wireless communications, vol. 11, no. 5, pp. 1676-1687, May, 2012. Article (CrossRef Link).

[22] C. Wang, T. Liu and X. Dong, "Impact of channel estimation error on the performance of amplify-and-forward two-way relaying," IEEE Transactions on vehicular technology, vol. 61, no. 3, pp. 1197-1207, March, 2012. Article (CrossRef Link).

[23] M.K. Arti and R.B. Manav. "Performance analysis of two-way AF MIMO Relaying of OSTBCs with imperfect channel gains," IEEE Transactions on vehicular technology, vol. 63, no. 8, pp. 4118-4124, October, 2014. Article (CrossRef Link).

[25] N. N. Tran, D. H. Pham, H. D. Tuan and H. Nguyen. "Orthogonal affine precoding and decoding for channel estimation and source detection in MIMO frequency-selective fading channel,” IEEE Transactions on signal processing, vol.57, no. 3, pp. 1151-1162, March, 2009. Article (CrossRef Link).

[26] A. Vosoughi, A. Scaglione. "Everything you always wanted to know about training: guidelines derived using the affine precoding framework and CRB," IEEE Transactions on Signal Processing, vol. 54, no. 3, pp. 940-954, March, 2006. Article (CrossRef Link). 


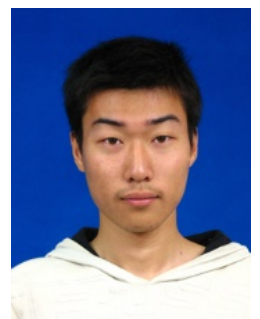

Xianwen He is now a doctoral candidate in Naval University of Engineering, China. He received the B.S. and M.S. degree from University of Electronic Science and Technology of China (UESTC) and Naval University of Engineering in 2012 and 2015 respectively. His research interests are in the general area of cooperative wireless communication.

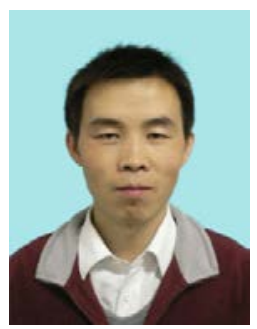

Gaoqi Dou received B.S. and Ph.D. degree from the Department of communication Engineering, Naval University of Engineering, China, in 2004 and 2009. He is currently an associate professor at the Department of Communication Engineering. His research interests are in the general area of wireless channel estimation and equalization and iterative processing.

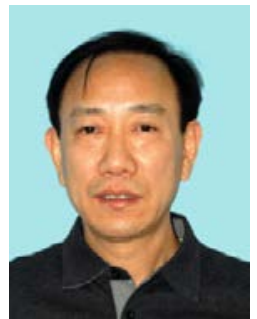

Jun Gao received the M.S. and Ph.D. degrees from Beijing Institute of Technology (BIT), in 1986 and 1989 respectively. He is currently a professor at the Department of Communication Engineering. His research interests are in the general area of wireless communication, with emphasis on digital communication and its applications. 